\title{
THE DAUGAVET PROPERTY FOR SPACES OF LIPSCHITZ FUNCTIONS
}

\author{
YEVGEN IVAKHNO, VLADIMIR KADETS and DIRK WERNER*
}

\begin{abstract}
For a compact metric space $K$ the space $\operatorname{Lip}(K)$ has the Daugavet property if and only if the norm of every $f \in \operatorname{Lip}(K)$ is attained locally. If $K$ is a subset of an $L_{p}$-space, $1<p<\infty$, this is equivalent to the convexity of $K$.
\end{abstract}

\section{Introduction}

A Banach space $X$ is said to have the Daugavet property if

$$
\|\mathrm{Id}+T\|=1+\|T\|
$$

for every rank-1 operator $T: X \rightarrow X$; then (1.1) also holds for all weakly compact operators on $X$ and even all operators that do not fix copies of $\ell_{1}$. The Daugavet property was introduced in [5] and further studied in [10] and [6], but examples of spaces having the Daugavet property have long been known; e.g., $C[0,1], L_{1}[0,1], L_{\infty}[0,1]$, the disk algebra, $H^{\infty}$, etc.

In this paper we shall investigate the Daugavet property for spaces of Lipschitz functions. Throughout, $(K, \rho)$ stands for a complete metric space that is not reduced to a singleton. The space of all Lipschitz functions on $K$ will be equipped with the seminorm

$$
\|f\|=\sup \left\{\frac{\left|f\left(t_{1}\right)-f\left(t_{2}\right)\right|}{\rho\left(t_{1}, t_{2}\right)}: t_{1} \neq t_{2} \in K\right\} .
$$

If one quotients out the kernel of this seminorm, i.e., the constant functions, one obtains the Banach space $\operatorname{Lip}(K)$, whose norm will also be denoted by $\|\cdot\|$. Equivalently, one can fix a point $t_{0} \in K$ and consider the Banach space $\operatorname{Lip}_{0}(K)$ consisting of all Lipschitz functions on $K$ that vanish at $t_{0}$, with the Lipschitz constant as an actual norm. It is easily seen that $\operatorname{Lip}(K)$ and $\operatorname{Lip}_{0}(K)$

\footnotetext{
* The work of the second-named author was supported by a fellowship from the Alexandervon-Humboldt Stiftung.

Received January 31, 2006.
} 
are isometrically isomorphic. In this paper we prefer the first point of view, but will refer to the elements of $\operatorname{Lip}(K)$ as functions rather than equivalence classes, as is familiar with $L_{p}$-spaces.

Since $\operatorname{Lip}[0,1]$ is isometric to $L_{\infty}[0,1]$ via differentiation almost everywhere, it is clear that $\operatorname{Lip}[0,1]$ has the Daugavet property. On the other hand the Hölder space $H^{\alpha}[0,1]$, being the dual of a space with the RNP [13, p. 83], fails the Daugavet property by the results of [16]; $H^{\alpha}[0,1]$ is just the Lipschitz space for $K=[0,1]$ with the metric $\rho_{\alpha}(s, t)=|s-t|^{\alpha}$. But for the unit square $Q=[0,1] \times[0,1]$ with the Euclidean metric it is far from obvious whether the Daugavet property holds for $\operatorname{Lip}(Q)$; in fact, this will turn out to be true as a special case of Theorem 3.1 below. The validity of the Daugavet property of $\operatorname{Lip}(Q)$ was asked in [15].

Whereas for the "classical" function spaces the validity of the Daugavet property is equivalent to a nonatomicity condition ([3] for $C(S)$ and $L_{1}(\mu)$, [16] for function algebras, [14] for $L_{1}$-preduals and [8] for the noncommutative case), in the setting of Lipschitz spaces it is a locality condition that plays a similar role, for in Theorem 3.3 we will show for a compact metric space $K$ that the Daugavet property of $\operatorname{Lip}(K)$ is equivalent to the fact that every Lipschitz function on $K$ almost attains its norm at close-by points; see Definition 2.2(a) for precision. We also characterise compact "local" metric spaces by a condition that is reminiscent of metric convexity (Proposition 2.8) and is sometimes even equivalent to it, e.g., for compact subsets of $L_{p}, 1<p<\infty$ (Proposition 2.9). As a result, for a compact subset of $L_{p}, 1<p<\infty$, the Daugavet property of $\operatorname{Lip}(K)$ is equivalent to the convexity of $K$.

An important tool to construct Lipschitz functions is McShane's extension theorem saying that if $M \subset K$ and $f: M \rightarrow \mathrm{R}$ is a Lipschitz function, then there is an extension to a Lipschitz function $F: K \rightarrow \mathrm{R}$ with the same Lipschitz constant; see [1, p. 12/13]. This will be used several times.

We will also make use of the following geometric characterisations of the Daugavet property from [5] and [2]. Part (iii) is particularly useful when one doesn't have full access to the dual space. As for notation, we denote the closed unit ball (resp. sphere) of a Banach space $X$ by $B_{X}$ (resp. $S_{X}$ ) and the closed ball with centre $t$ and radius $r$ in a metric space $K$ by $B_{K}(t, r)$.

LeMma 1.1. The following assertions are equivalent:

(i) $X$ has the Daugavet property.

(ii) For every $y \in S_{X}, x^{*} \in S_{X^{*}}$ and $\varepsilon>0$ there exists some $x \in S_{X}$ such that $x^{*}(x) \geq 1-\varepsilon$ and $\|x+y\| \geq 2-\varepsilon$.

(iii) For every $\varepsilon>0$ and for every $y \in S_{X}$ the closed convex hull of the set $\left\{u \in(1+\varepsilon) B_{X}:\|y+u\| \geq 2-\varepsilon\right\}$ contains $S_{X}$. 


\section{Local metric spaces}

Let us recall that a metric space $K$ is called metrically convex if for any two points $t_{1}, t_{2} \in K$ two closed balls $B_{K}\left(t_{1}, r_{1}\right)$ and $B_{K}\left(t_{2}, r_{2}\right)$ intersect if and only if $\rho\left(t_{1}, t_{2}\right) \leq r_{1}+r_{2}$.

Clearly, convex subsets of normed spaces are metrically convex, and $S^{1}=$ $\left\{(x, y) \in \mathbf{R}^{2}: x^{2}+y^{2}=1\right\}$ is metrically convex for the geodesic metric, but not for the Euclidean metric.

We shall need the following lemma.

Lemma 2.1. A complete metric space $K$ is metrically convex if and only if for every two distinct points $t, \tau \in K$ there is an isometric embedding $\phi:[0, a] \rightarrow K$ (where $a=\rho(t, \tau)$ ) such that $\phi(0)=t, \phi(a)=\tau$. In other words, $K$ is metrically convex if and only if every two points of $K$ can be connected by an isometric copy of a linear segment.

Proof. The property displayed in the lemma clearly implies the metric convexity of $K$. To prove the converse, let $K$ be metrically convex and let $t$ and $\tau$ be two points at a distance $a$; we shall label them $t_{0}$ and $t_{a}$. Then there is a point $t_{a / 2} \in B_{K}\left(t_{0}, a / 2\right) \cap B_{K}\left(t_{a}, a / 2\right)$. It follows that $\rho\left(t_{0}, t_{a / 2}\right)=\rho\left(t_{a / 2}, t_{a}\right)=$ $a / 2$. Likewise, pick points $t_{a / 4} \in B_{K}\left(t_{0}, a / 4\right) \cap B_{K}\left(t_{a / 2}, a / 4\right)$ and $t_{3 / 4 \cdot a} \in$ $B_{K}\left(t_{a / 2}, a / 4\right) \cap B_{K}\left(t_{a}, a / 4\right)$. Continuing in this manner, one obtains for each dyadic rational $d \in[0,1]$ a point $t_{d a} \in K$ such that $\rho\left(t_{d a}, t_{d^{\prime} a}\right)=\left|d-d^{\prime}\right| a$. The mapping $d a \mapsto t_{d a}$ can now be extended to an isometric mapping $\phi:[0, a] \rightarrow$ $K$, as requested.

The following definition is crucial for this paper.

Definition 2.2. Let $K$ be a metric space.

(a) The space $K$ is called local if for every $\varepsilon>0$ and for every function $f \in$ $\operatorname{Lip}(K)$ there are two distinct points $\tau_{1}, \tau_{2} \in K$ such that $\rho\left(\tau_{1}, \tau_{2}\right)<\varepsilon$ and

$$
\frac{f\left(\tau_{2}\right)-f\left(\tau_{1}\right)}{\rho\left(\tau_{1}, \tau_{2}\right)}>\|f\|-\varepsilon .
$$

(b) Let $f \in \operatorname{Lip}(K)$ and $\varepsilon>0$. A point $t \in K$ is said to be an $\varepsilon$-point of $f$ if in every neighbourhood $U \subset K$ of $t$ there are two points $\tau_{1}, \tau_{2} \in U$ for which (2.1) holds true.

(c) The space $K$ is called spreadingly local if for every $\varepsilon>0$ and for every function $f \in \operatorname{Lip}(K)$ there are infinitely many $\varepsilon$-points of $f$.

The next proposition provides a large class of examples. 
Proposition 2.3. A metrically convex complete metric space $K$ is spreadingly local.

Proof. Fix an $\varepsilon>0$ and a function $f \in \operatorname{Lip}(K)$ with $\|f\|=1$. Select $t, \tau \in K$ with $\rho(t, \tau)>0$ such that

$$
f(\tau)-f(t)>(1-\varepsilon) \rho(t, \tau) .
$$

Denote $a=\rho(t, \tau)$ and apply Lemma 2.1 to this pair of points. The function $F=f \circ \phi:[0, a] \rightarrow \mathrm{R}$, where $\phi$ is from Lemma 2.1, is 1-Lipschitz. Hence $\left|F^{\prime}\right| \leq 1$ a.e. on $[0, a]$ and

$$
\int_{0}^{a} F^{\prime}(r) d r=f(\tau)-f(t)>(1-\varepsilon) a .
$$

Therefore there are infinitely many points $r_{i} \in[0, a]$ with $F^{\prime}\left(r_{i}\right)>1-\varepsilon$. Let us show that every point of the form $t_{i}=\phi\left(r_{i}\right)$ is an $\varepsilon$-point of $f$. By the definition of the derivative we have

$$
\frac{F\left(r_{i}+\delta_{i}\right)-F\left(r_{i}\right)}{\delta_{i}}>1-\varepsilon
$$

for sufficiently small $\delta_{i} \in(0, \varepsilon)$. Denote $\tau_{i}=\phi\left(r_{i}+\delta_{i}\right)$. Then $\rho\left(t_{i}, \tau_{i}\right)=\delta_{i}$ and $f\left(\tau_{i}\right)-f\left(t_{i}\right)>(1-\varepsilon) \delta_{i}$.

Actually this proposition applies to a slightly more general class of spaces $K$, defined by the requirement that for each pair of points $t, \tau \in K$ and each $\eta>0$ there exists a curve of length $\leq \rho(t, \tau)+\eta=: a_{\eta}$ joining $t$ and $\tau$. In other words, there exists a 1-Lipschitz mapping (having arclength as parameter) $\phi:\left[0, a_{\eta}\right] \rightarrow K$ with $\phi(0)=t, \phi\left(a_{\eta}\right)=\tau$. Such spaces could be termed almost metrically convex. A variant of the above proof then shows that almost metrically convex spaces are spreadingly local.

EXAMPLE 2.4. There is a (noncompact) almost metrically convex space that is not metrically convex. Indeed, let

$$
M=\left\{f \in L_{1}[0,1]:|f|=1 \text { a.e. }\right\} ;
$$

this is a closed subset of $L_{1}$. Instead of the $L_{1}$-norm we shall use the following equivalent norm on $L_{1}$. Pick a total sequence of functionals $x_{n}^{*} \in S_{L_{\infty}}$ and put, for $f \in L_{1}$,

$$
\||f|\|=\|f\|_{L_{1}}+\left(\sum_{n=1}^{\infty} 2^{-n}\left|x_{n}^{*}(f)\right|^{2}\right)^{1 / 2} .
$$

This norm is strictly convex. It follows that $M$, equipped with the metric $\rho(f, g)=\|\| f-g \|$, is not metrically convex since it is not convex; indeed, 
if $f, g \in M$, then $n o$ nontrivial convex combination belongs to $M$ (unless $f=g$ ).

On the other hand, $(M, \rho)$ is almost metrically convex. To see this let $f \neq g$ be two functions in $M$. For a Borel set $A \subset[0,1]$ define $h_{A} \in M$ by

$$
h_{A}=f \chi_{A}+g \chi_{[0,1] \backslash A} .
$$

Given $\varepsilon>0$, pick $\varepsilon^{\prime} \leq \varepsilon\|f-g\|$ and $N \in \mathbf{N}$ such that $2\left(\sum_{n>N} 2^{-n}\right)^{1 / 2} \leq \varepsilon^{\prime}$. Define a nonatomic vector measure taking values in $\mathrm{R}^{N+1}$ by

$$
\mu(A)=\left(\int_{A}|f-g|, x_{1}^{*}\left((f-g) \chi_{A}\right), \ldots, x_{N}^{*}\left((f-g) \chi_{A}\right)\right) .
$$

By the Lyapunov convexity theorem [9, Th. 5.5] there exists a Borel set $\Delta$ such that $\mu(\Delta)=\frac{1}{2} \mu([0,1])$. We then have, since $g-h_{\Delta}=(g-f) \chi_{\Delta}$

$$
\begin{aligned}
\left\|g-h_{\Delta}\right\| & =\left\|g-h_{\Delta}\right\|_{L_{1}}+\left(\sum_{n=1}^{\infty} 2^{-n}\left|x_{n}^{*}\left(g-h_{\Delta}\right)\right|^{2}\right)^{1 / 2} \\
& \leq \int_{\Delta}|f-g|+\left(\sum_{n=1}^{N} 2^{-n}\left|x_{n}^{*}\left((f-g) \chi_{\Delta}\right)\right|^{2}\right)^{1 / 2}+\varepsilon^{\prime} \\
& =\frac{1}{2} \int_{0}^{1}|f-g|+\frac{1}{2}\left(\sum_{n=1}^{N} 2^{-n}\left|x_{n}^{*}(f-g)\right|^{2}\right)^{1 / 2}+\varepsilon^{\prime} \\
& \leq \frac{1}{2}|\|f-g\||+\varepsilon^{\prime} \leq\left(\frac{1}{2}+\varepsilon\right)\|f-g\| \mid
\end{aligned}
$$

and likewise

$$
\left\|f-h_{\Delta}\right\| \leq\left(\frac{1}{2}+\varepsilon\right)\|f-g\| .
$$

Let $F_{0}=f, F_{1}=g, F_{1 / 2}=h_{\Delta}$. Now we reiterate the above construction, first applying it to $F_{0}, F_{1 / 2}$ and $\varepsilon / 2$ and then to $F_{1 / 2}, F_{1}$ and $\varepsilon / 2$ to obtain functions $F_{1 / 4}, F_{3 / 4} \in M$ such that

$$
\begin{aligned}
& \max \left\{\left\|F_{0}-F_{1 / 4}\right\|,\left\|F_{1 / 2}-F_{1 / 4}\right\|\right\} \leq\left(\frac{1}{2}+\frac{\varepsilon}{2}\right)\left\|F_{0}-F_{1 / 2}\right\|, \\
& \max \left\{\left\|F_{1 / 2}-F_{3 / 4}\right\|\|,\| F_{1}-F_{1 / 4} \|\right\} \leq\left(\frac{1}{2}+\frac{\varepsilon}{2}\right)\left\|F_{1}-F_{1 / 2}\right\| .
\end{aligned}
$$

Continuing in this manner, we can assign to each dyadic rational $d \in[0,1]$ a function $F_{d} \in M$ such that the curve $[0,1] \rightarrow M, t \mapsto F_{t}$, obtained from this 
by continuous extension, has a length that can be estimated from above by $\sup _{n}\left(\frac{1}{2}+\frac{\varepsilon}{2^{n-1}}\right)\left(\frac{1}{2}+\frac{\varepsilon}{2^{n-2}}\right) \cdots\left(\frac{1}{2}+\varepsilon\right) 2^{n} \leq \exp \left(2^{2-n} \varepsilon+\cdots+2 \varepsilon\right) \leq e^{4 \varepsilon}$.

Therefore $M$ is almost metrically convex.

We will need a lemma in order to control the Lipschitz constant of a function by the Lipschitz constant of some restriction under highly technical assumptions that we shall meet later. In the following, $\sqcup$ is used to indicate a disjoint union.

Lemma 2.5. Let $A=B \sqcup C$ be a metric space, $r \in(0,1 / 4], \delta<r^{2} / 16$, $\rho(B, C)>r$. Suppose $\tilde{C} \subset C$ is a $\delta$-net of $C$ such that every two points of $\tilde{C}$ are at least $r$-distant, and let $f: A \rightarrow \mathrm{R}$ be a function that is 1-Lipschitz on $B \sqcup \tilde{C}$ and also 1-Lipschitz on every ball $B_{A}(t, \delta)$ for $t \in \tilde{C}$. Then $f$ is $(1+r / 2)$-Lipschitz on the whole space A.

Proof. Consider arbitrary points $s_{1} \neq s_{2} \in A$. We have to prove that

$$
\left|\frac{f\left(s_{2}\right)-f\left(s_{1}\right)}{\rho\left(s_{1}, s_{2}\right)}\right| \leq 1+r / 2 .
$$

We have to distinguish three cases: firstly, when $s_{1}, s_{2} \in B$; secondly, when $s_{1}, s_{2} \in C$; and thirdly, when one of the points (say, $s_{1}$ ) belongs to $B$ and the other belongs to $C$.

In the first case (2.2) holds true even with 1 on the right hand side by assumption on $f$. Consider the second case. If $s_{1}, s_{2}$ belong to the same ball of the form $B_{A}(t, \delta)$ for $t \in \tilde{C}$, then the job is likewise done. If not, let $t_{1} \neq t_{2} \in \tilde{C}$ be points such that $\rho\left(t_{1}, s_{1}\right) \leq \delta$ and $\rho\left(t_{2}, s_{2}\right) \leq \delta$. Then

$$
\begin{aligned}
\left|\frac{f\left(s_{2}\right)-f\left(s_{1}\right)}{\rho\left(s_{1}, s_{2}\right)}\right| & \leq\left|\frac{f\left(s_{2}\right)-f\left(t_{2}\right)}{\rho\left(s_{1}, s_{2}\right)}\right|+\left|\frac{f\left(t_{2}\right)-f\left(t_{1}\right)}{\rho\left(s_{1}, s_{2}\right)}\right|+\left|\frac{f\left(t_{1}\right)-f\left(s_{1}\right)}{\rho\left(s_{1}, s_{2}\right)}\right| \\
& \leq \frac{\delta}{\rho\left(s_{1}, s_{2}\right)}+\frac{\rho\left(t_{2}, t_{1}\right)}{\rho\left(s_{1}, s_{2}\right)}+\frac{\delta}{\rho\left(s_{1}, s_{2}\right)} \\
& \leq \frac{2 \delta}{r-2 \delta}+\frac{\rho\left(t_{2}, t_{1}\right)}{\rho\left(t_{2}, t_{1}\right)-2 \delta} \\
& \leq \frac{2 \delta}{r-2 \delta}+1+\frac{2 \delta}{\rho\left(t_{2}, t_{1}\right)-2 \delta} \\
& \leq 1+\frac{4 \delta}{r-2 \delta} \leq 1+r / 2 .
\end{aligned}
$$


In the last case find $t_{2} \in \tilde{C}$ such that $\rho\left(t_{2}, s_{2}\right) \leq \delta$. Then

$$
\begin{aligned}
\left|\frac{f\left(s_{2}\right)-f\left(s_{1}\right)}{\rho\left(s_{1}, s_{2}\right)}\right| & \leq\left|\frac{f\left(s_{2}\right)-f\left(t_{2}\right)}{\rho\left(s_{1}, s_{2}\right)}\right|+\left|\frac{f\left(t_{2}\right)-f\left(s_{1}\right)}{\rho\left(s_{1}, s_{2}\right)}\right| \\
& \leq \frac{\delta}{\rho\left(s_{1}, s_{2}\right)}+\frac{\rho\left(t_{2}, s_{1}\right)}{\rho\left(s_{1}, s_{2}\right)} \\
& \leq \frac{\delta}{r}+\frac{\rho\left(t_{2}, s_{1}\right)}{\rho\left(t_{2}, s_{1}\right)-\delta} \\
& \leq \frac{\delta}{r}+\frac{r}{r-\delta}=1+\frac{\delta}{r}+\frac{\delta}{r-\delta} \leq 1+r / 2 .
\end{aligned}
$$

This completes the proof of the lemma.

Obviously, a spreadingly local space is local. In the compact case the converse is valid, too, as will be pointed out now.

LEMMA 2.6. If $K$ is compact and local, then it is spreadingly local.

Proof. We will prove by induction on $n$ that for every $f \in \operatorname{Lip}(K)$ and for every $\varepsilon>0$ there are $n \varepsilon$-points of $f$.

Thanks to the compactness of $K$ every function $f \in \operatorname{Lip}(K)$ has a " 0 point", i.e., a point that is an $\varepsilon$-point for every $\varepsilon>0$. Indeed, take a sequence of pairs $t_{n}, \tau_{n} \in K$ satisfying Definition 2.2 with $\varepsilon=1 / n, n=1,2, \ldots$, and take an arbitrary limit point of $\left(t_{n}\right)$. So the start of the induction holds true. Now assume the statement for a fixed $n$ and let us prove it for $n+1$.

Take an $f \in \operatorname{Lip}(K)$ with $\|f\|=1$ and $\varepsilon \in(0,1 / 4]$. Due to our hypothesis there are $\varepsilon$-points $t_{1}, \ldots, t_{n}$ of $f$. Also, select two points $\tau_{1}, \tau_{2} \in K$ distinct from all the $t_{i}$ and such that

$$
\frac{f\left(\tau_{2}\right)-f\left(\tau_{1}\right)}{\rho\left(\tau_{1}, \tau_{2}\right)}>1-\varepsilon / 4 .
$$

Let $r \in(0, \varepsilon / 4]$ be a number so small that the balls $U_{i}=B_{K}\left(t_{i}, r\right), i=$ $1, \ldots, n$, are disjoint and contain neither $\tau_{1}$ nor $\tau_{2}$. Fix a $\delta<r^{2} / 16$, denote the interior of $B_{K}\left(t_{i}, \delta\right)$ by $V_{i}$ and consider $\tilde{K}=\left(K \backslash \bigcup_{i=1}^{n} U_{i}\right) \sqcup \bigcup_{i=1}^{n} V_{i}$ as a subspace of the metric space $K$. Define $\tilde{f}: \tilde{K} \rightarrow \mathrm{R}$ as follows: $\tilde{f}(t)=f(t)$ for $t \in K \backslash \bigcup_{i=1}^{n} U_{i}$ and $\tilde{f}(t)=f\left(t_{i}\right)$ on the corresponding $V_{i}$. Lemma 2.5 implies that $\tilde{f}$ satisfies a Lipschitz condition on $\tilde{K}$ with the constant $1+\varepsilon / 2$. Extend $\tilde{f}$ to a function on $K$ preserving the Lipschitz constant, still denoted by $\tilde{f}$.

Take as $t_{n+1}$ an arbitrary 0 -point of the function $g=f+\tilde{f}$. Since

$$
\|g\| \geq \frac{g\left(\tau_{2}\right)-g\left(\tau_{1}\right)}{\rho\left(\tau_{1}, \tau_{2}\right)}=2 \frac{f\left(\tau_{2}\right)-f\left(\tau_{1}\right)}{\rho\left(\tau_{1}, \tau_{2}\right)}>2-\varepsilon / 2,
$$


in every neighbourhood of $t_{n+1}$ there are points $s_{1}, s_{2}$ with

$$
\frac{f\left(s_{2}\right)-f\left(s_{1}\right)}{\rho\left(s_{1}, s_{2}\right)}+\frac{\tilde{f}\left(s_{2}\right)-\tilde{f}\left(s_{1}\right)}{\rho\left(s_{1}, s_{2}\right)}>2-\varepsilon / 2 .
$$

This implies that $t_{n+1}$ cannot belong to any $V_{i}$ since in $V_{i}$ the second fraction of (2.3) is zero, but the first one is not greater than 1 ; hence $t_{n+1}$ differs from all the other $t_{i}$. On the other hand, by our construction $\|\tilde{f}\| \leq 1+\varepsilon / 2$, so the second fraction of (2.3) is $\leq 1+\varepsilon / 2$. Hence there is an estimate for the first fraction, namely

$$
\frac{f\left(s_{2}\right)-f\left(s_{1}\right)}{\rho\left(s_{1}, s_{2}\right)}>1-\varepsilon,
$$

which means that $t_{n+1}$ is an $\varepsilon$-point of $f$.

Next we are going to characterise local metric spaces intrinsically, at least in the compact case, using the following geometric property that we have chosen to give an ad-hoc name.

Definition 2.7. A metric space $K$ has property $(Z)$ if the following condition is met: Given $t, \tau \in K$ and $\varepsilon>0$, there is some $z \in K \backslash\{t, \tau\}$ satisfying

$$
\rho(t, z)+\rho(z, \tau) \leq \rho(t, \tau)+\varepsilon \min \{\rho(z, t), \rho(z, \tau)\} .
$$

A compact space satisfying (2.4) with $\varepsilon=0$ is easily seen to be metrically convex. Thus, property $(Z)$ is " $\varepsilon$-close" to metric convexity, and there are instances when $(Z)$ actually implies metric convexity; see Corollary 2.10 and Remark 2.11 below.

Here is the connection between locality and property $(Z)$.

Proposition 2.8. Let $K$ be a metric space.

(a) If $K$ is local, then $K$ has property $(Z)$.

(a) If $K$ is compact and has property ( $Z$ ), then $K$ is local.

Proof. (a) Assume that $K$ fails property (Z), i.e., for some $t_{0}, \tau_{0} \in K$ and $\varepsilon_{0}>0$ there are no points $z \in K \backslash\left\{t_{0}, \tau_{0}\right\}$ as in (2.4). For a point $z \in K$ let $r(z)=\rho\left(z, t_{0}\right), s(z)=\rho\left(z, \tau_{0}\right)$ and $d=\rho\left(t_{0}, \tau_{0}\right)$. Pick $\varepsilon>0$ with

$$
\frac{\varepsilon}{1-\varepsilon}<\frac{\varepsilon_{0}}{4} \text {. }
$$


Now define $f: K \rightarrow \mathrm{R}$ by

$$
f(z)=\left\{\begin{aligned}
\max \{d / 2-(1-\varepsilon) s(z), 0\} & \\
& \text { if } r(z) \geq s(z), r(z)+(1-2 \varepsilon) s(z) \geq d \\
-\max \{d / 2-(1-\varepsilon) & r(z), 0\} \\
& \text { if } r(z) \leq s(z),(1-2 \varepsilon) r(z)+s(z) \geq d
\end{aligned}\right.
$$

This function is well defined, since for $r(z)=s(z)$ both parts of the definition yield 0 , and all points of $K$ are covered in the two "if" cases by our assumption on $K$; note that $2 \varepsilon<\varepsilon_{0}$.

Let us show that $f$ is a Lipschitz function with $\|f\|=1$. Indeed, the only critical case is to estimate $f\left(z_{2}\right)-f\left(z_{1}\right)$ when $f\left(z_{2}\right)>0$ and $f\left(z_{1}\right)<0$; in this case

$$
\begin{aligned}
f\left(z_{2}\right)-f\left(z_{1}\right)= & \left(\frac{d}{2}-(1-\varepsilon) s\left(z_{2}\right)\right)+\left(\frac{d}{2}-(1-\varepsilon) r\left(z_{1}\right)\right) \\
\leq & \left(\frac{r\left(z_{2}\right)+(1-2 \varepsilon) s\left(z_{2}\right)}{2}-(1-\varepsilon) s\left(z_{2}\right)\right) \\
& \quad+\left(\frac{(1-2 \varepsilon) r\left(z_{1}\right)+s\left(z_{1}\right)}{2}-(1-\varepsilon) r\left(z_{1}\right)\right) \\
= & \frac{1}{2}\left(r\left(z_{2}\right)-s\left(z_{2}\right)\right)+\frac{1}{2}\left(s\left(z_{1}\right)-r\left(z_{1}\right)\right) \\
\leq & \rho\left(z_{1}, z_{2}\right) ;
\end{aligned}
$$

also, the norm is attained at $\tau_{0}, t_{0}$, i.e., $f\left(\tau_{0}\right)-f\left(t_{0}\right)=\rho\left(\tau_{0}, t_{0}\right)$.

Consider now points $z_{1}, z_{2} \in K$ where

$$
\frac{f\left(z_{2}\right)-f\left(z_{1}\right)}{\rho\left(z_{2}, z_{1}\right)}>1-\varepsilon ;
$$

we shall show that then $z_{1}$ is close to $t_{0}$ and $z_{2}$ is close to $\tau_{0}$ so that their distance is necessarily big. Obviously, we must have $f\left(z_{2}\right)>0$ and $f\left(z_{1}\right)<0$ for $(2.5)$ to subsist. In particular, we have

$$
\rho\left(z_{1}, t_{0}\right)<\rho\left(z_{1}, \tau_{0}\right) ; \quad \rho\left(z_{2}, \tau_{0}\right)<\rho\left(z_{2}, t_{0}\right) .
$$


Hence

$$
\begin{aligned}
(1-\varepsilon) \rho\left(z_{1}, z_{2}\right) & <f\left(z_{2}\right)-f\left(z_{1}\right) \\
& =\left(\frac{d}{2}-(1-\varepsilon) \rho\left(z_{2}, \tau_{0}\right)\right)-\left(\frac{d}{2}-(1-\varepsilon) \rho\left(z_{1}, t_{0}\right)\right) \\
& =d-(1-\varepsilon)\left(\rho\left(z_{2}, \tau_{0}\right)+\rho\left(z_{1}, t_{0}\right)\right) ;
\end{aligned}
$$

in other words

$$
(1-\varepsilon)\left(\rho\left(z_{1}, z_{2}\right)+\rho\left(z_{2}, \tau_{0}\right)+\rho\left(z_{1}, t_{0}\right)\right)<d
$$

so that

$$
\rho\left(z_{k}, t_{0}\right)+\rho\left(z_{k}, \tau_{0}\right)<\frac{d}{1-\varepsilon}, \quad k=1,2 .
$$

By our choice of $\varepsilon_{0}, t_{0}, \tau_{0}$ and (2.6)

$$
\rho\left(z_{1}, t_{0}\right)+\rho\left(z_{1}, \tau_{0}\right) \geq d+\varepsilon_{0} \rho\left(z_{1}, t_{0}\right)
$$

so that by $(2.7)$

$$
d+\varepsilon_{0} \rho\left(z_{1}, t_{0}\right)<\frac{d}{1-\varepsilon}
$$

and hence $\rho\left(z_{1}, t_{0}\right)<d / 4$ by our choice of $\varepsilon$. Likewise $\rho\left(z_{2}, \tau_{0}\right)<d / 4$ and consequently $\rho\left(z_{1}, z_{2}\right)>d / 2$. Therefore, $K$ cannot be local.

(b) Assume that $K$ is not local. Then there is a Lipschitz function $f$ with $\|f\|=1$ for which (2.1) is impossible for $\tau_{1}, \tau_{2}$ at small distance, viz. for $\rho\left(\tau_{1}, \tau_{2}\right)<\varepsilon$. By a compactness argument one hence deduces the existence of points $t, \tau \in K$ such that

$$
\frac{f(\tau)-f(t)}{\rho(\tau, t)}=1
$$

and $\rho(t, \tau)$ is minimal among all points as in (2.8). Now let $\varepsilon_{n} \searrow 0$ and apply condition $(Z)$ to $t, \tau$ and $\varepsilon_{n}$. This yields a sequence of points $z_{n} \in K \backslash\{t, \tau\}$ such that

$$
\rho\left(t, z_{n}\right)+\rho\left(z_{n}, \tau\right) \leq \rho(t, \tau)+\varepsilon_{n} \min \left\{\rho\left(z_{n}, t\right), \rho\left(z_{n}, \tau\right)\right\}
$$

Passing to a subsequence we may assume that $\left(z_{n}\right)$ converges, say $z_{n} \rightarrow z_{0}$, and that without loss of generality

$$
\rho\left(t, z_{n}\right) \leq \rho\left(\tau, z_{n}\right) \quad \forall n \geq 1
$$


Note that

$$
\rho\left(t, z_{0}\right)+\rho\left(z_{0}, \tau\right)=\rho(t, \tau) .
$$

If $z_{0} \neq t$, then

$$
\begin{aligned}
1 \geq \frac{f\left(z_{0}\right)-f(t)}{\rho\left(z_{0}, t\right)} & =\frac{f(\tau)-f(t)}{\rho(\tau, t)} \frac{\rho(\tau, t)}{\rho\left(z_{0}, t\right)}-\frac{f(\tau)-f\left(z_{0}\right)}{\rho\left(\tau, z_{0}\right)} \frac{\rho\left(z_{0}, \tau\right)}{\rho\left(z_{0}, t\right)} \\
& \geq \frac{\rho(\tau, t)}{\rho\left(z_{0}, t\right)}-\frac{\rho\left(z_{0}, \tau\right)}{\rho\left(z_{0}, t\right)}=1
\end{aligned}
$$

by (2.11), and thus $f$ attains its norm at the pair $z_{0}, t$. But by (2.10)

$$
\rho\left(t, z_{0}\right) \leq \frac{1}{2}\left(\rho\left(t, z_{0}\right)+\rho\left(\tau, z_{0}\right)\right)=\frac{1}{2} \rho(t, \tau),
$$

which contradicts the minimality condition imposed on the pair $t, \tau$.

Therefore, $z_{n} \rightarrow t$, and for sufficiently large $n$ we have $\rho\left(t, z_{n}\right)<\varepsilon$ along with (2.9). But then

$$
\begin{aligned}
\frac{f\left(z_{n}\right)-f(t)}{\rho\left(z_{n}, t\right)} & =\frac{f(\tau)-f(t)}{\rho(\tau, t)} \frac{\rho(\tau, t)}{\rho\left(t, z_{n}\right)}-\frac{f(\tau)-f\left(z_{n}\right)}{\rho\left(\tau, z_{n}\right)} \frac{\rho\left(\tau, z_{n}\right)}{\rho\left(t, z_{n}\right)} \\
& \geq \frac{\rho(\tau, t)-\rho\left(\tau, z_{n}\right)}{\rho\left(t, z_{n}\right)} \geq 1-\varepsilon
\end{aligned}
$$

by (2.9), which contradicts our choice of $f$, since $\rho\left(t, z_{n}\right)<\varepsilon$.

The definition of locality immediately implies that a compact local space is connected; one just has to apply the definition with the indicator function of a set that is both open and closed. We will now present a class of compact metric spaces for which property $(Z)$ and hence locality implies (metric) convexity. Recall that a Banach space $\left(E,\|\|_{E}\right)$ is called locally uniformly rotund if for each $x \in S_{E}$ and $\eta>0$ there is some $\delta=\delta_{x}(\eta)>0$ such that $\|x-y\|_{E} \leq \eta$ whenever $y \in B_{E}$ and $\left\|\frac{1}{2}(x+y)\right\|_{E} \geq 1-\delta$.

Proposition 2.9. Let $\left(E,\|\cdot\|_{E}\right)$ be a smooth locally uniformly rotund Banach space and let $K \subset E$ be a compact subset with property (Z). Then $K$ is convex.

Proof. By a result of Vlasov ([12], [11, Th. 2.2, p. 368]) a compact Chebyshev subset of a smooth Banach space is convex. If we assume that $K$ is not convex, this means that there are two points $P, Q \in K$ and a ball $B$ whose interior does not intersect $K$ with $P, Q \in \partial B$; we may assume that $B$ is centred at the origin, $B=B_{E}(0, \alpha)$, and by scaling that $\|P-Q\|_{E}=1$. Applying condition $(Z)$ to $P, Q$ and an arbitrary $\varepsilon>0$ yields some $z=z(\varepsilon) \in K \backslash\{P, Q\}$ 
as in (2.4). We may as well assume that $z_{0}=\lim _{\varepsilon \rightarrow 0} z(\varepsilon)$ exists; $z_{0}$ lies on the line segment $[P, Q]$ by strict convexity of $E$. Thus $z_{0}=P$ or $z_{0}=Q$; without loss of generality let us assume the latter. Fix, for the time being, $\varepsilon$ and $z=z(\varepsilon)$ and put $r=\|z-Q\|_{E}(<1 / 2)$.

Now consider $Q(\lambda)=\lambda P+(1-\lambda) Q, 0 \leq \lambda \leq 1$. Let us estimate $\|z-Q(\lambda)\|_{E}$ in order to derive a contradiction. On the one hand we have, since $z \in K$ and thus $\|z\|_{E} \geq \alpha$,

$$
\|z-Q(\lambda)\|_{E} \geq\|z\|_{E}-\|Q(\lambda)\|_{E} \geq \alpha-\|Q(\lambda)\|_{E}=: \varphi(\lambda) .
$$

Now $\varphi$ is a concave function of $\lambda$ with $\varphi(0)=0$ and

$$
\varphi(1 / 2)=\alpha-\left\|\frac{1}{2}(P+Q)\right\|>0
$$

by strict convexity. Hence with $\sigma=2 \varphi(1 / 2)$

$$
\|z-Q(r)\|_{E} \geq \varphi(r) \geq \sigma r .
$$

On the other hand, (2.4) means that $z \in B_{E}(P, 1-r+\varepsilon r)$; therefore the point $w=\frac{1}{2}(z+Q(r))$ also belongs to this ball, but $w \notin$ int $B_{E}(Q, r-\varepsilon r)$. In other words,

$$
\left\|\frac{(Q-z)+(Q-Q(r))}{2}\right\|_{E}=\left\|Q-\frac{z+Q(r)}{2}\right\|_{E} \geq r-\varepsilon r .
$$

Specifically, let $\eta=\sigma / 2$ and $0<\varepsilon<\delta_{P-Q}(\eta)$. Then (2.13) and local uniform rotundity (note that $\left.(Q-z) / r,(Q-Q(r)) / r \in B_{E}\right)$ imply that

$$
\|z-Q(r)\|_{E} \leq r \eta<r \sigma
$$

contradicting (2.12).

Proposition 2.9 applies in particular to $L_{p}$-spaces for $1<p<\infty$ and most particularly to Hilbert spaces.

We can sum up the previous results as follows.

Corollary 2.10. Let $K$ be a compact metric space. Then the following are equivalent:

(1) $K$ is local;

(2) $K$ is spreadingly local;

(3) $K$ has property $(Z)$.

If $K$ is a subset of a smooth locally uniformly rotund Banach space, then a further equivalent condition is: 
(4) $K$ is convex.

Another link between locality and metric convexity is provided by the following technical remark.

REMARK 2.11. Let us say that $K$ satisfies $\left(Z^{\prime}\right)$ if in addition to (2.4) in Definition 2.7 we require that

$$
\rho(z, \tau) \leq \rho(z, t) .
$$

Since one can exchange the roles of $t$ and $\tau$ here, this means that there is one point as in (2.4) that is closer to $\tau$ than to $t$ and another one that is closer to $t$ than to $\tau$. It is then possible to show that $\left(Z^{\prime}\right)$ implies metric convexity for compact spaces; see below. Hence locality implies metric convexity for those compact metric spaces that are symmetric in the sense that for any two points in $K$ there is an isometry on $K$ swapping these two points.

To prove this remark, we rephrase property $\left(Z^{\prime}\right)$ by saying that for every $\varepsilon>0$ and every $t, \tau \in K$ there exists some $z \in K \backslash\{\tau\}$ such that

$$
\begin{aligned}
(1-\varepsilon) \rho(\tau, z)+\rho(t, z) & \leq \rho(t, \tau), \\
\rho(\tau, z) & \leq \rho(t, z) .
\end{aligned}
$$

The strategy of the proof will be to infer from this in the compact case that for every $\varepsilon>0$ and every $t, \tau \in K$ there exists some $z \in K$ for which (2.14) holds and

$$
\frac{1}{10} \rho(t, \tau) \leq \rho(\tau, z) \leq \frac{9}{10} \rho(t, \tau) .
$$

If we let $\varepsilon \rightarrow 0$ and consider a limit point $z_{0}$ of the $z=z(\varepsilon)$ satisfying (2.14) and (2.16), then we can be certain that $z_{0} \neq t$ and $z_{0} \neq \tau$, but

$$
\rho\left(t, z_{0}\right)+\rho\left(z_{0}, \tau\right)=\rho(t, \tau) .
$$

As remarked earlier this implies the metric convexity of the compact space $K$.

Let us now come to the details. Fix $t, \tau$ and $\varepsilon$; we may suppose that $\rho(t, \tau)=1$. Assume for a contradiction that we cannot achieve (2.14) and (2.16) simultaneously. Let

$$
K_{0}=\{z \in K: \text { (2.14) and (2.15) hold }\} .
$$

Since $K_{0} \neq\{\tau\}$ by property $\left(Z^{\prime}\right)$, there is some $u \in K_{0}$ such that $\rho(u, t)<1$, and therefore $\alpha:=\min \left\{\rho(z, t): z \in K_{0}\right\}$ is attained at some $u_{0} \in K_{0} \backslash\{\tau\}$. Then $(1-\varepsilon) \rho\left(\tau, u_{0}\right)+\rho\left(u_{0}, t\right) \leq 1$ by (2.14). Now define $0 \leq \tilde{\varepsilon} \leq \varepsilon$ by

$$
(1-\tilde{\varepsilon}) \rho\left(\tau, u_{0}\right)+\rho\left(u_{0}, t\right)=1 .
$$


If $\tilde{\varepsilon}=0$, we have already found a point as in (2.17), and we are done. So we assume that $\tilde{\varepsilon}>0$ in the sequel. Then we can apply (2.14) and (2.15), i.e., property ( $Z$ ), with $t, u_{0}$ and $\tilde{\varepsilon}$ in place of $t, \tau$ and $\varepsilon$. This yields some $\tilde{z} \in K \backslash\left\{u_{0}\right\}$ with

$$
\begin{aligned}
(1-\tilde{\varepsilon}) \rho\left(u_{0}, \tilde{z}\right)+\rho(t, \tilde{z}) & \leq \rho\left(t, u_{0}\right), \\
\rho\left(u_{0}, \tilde{z}\right) & \leq \rho(t, \tilde{z}) .
\end{aligned}
$$

Next, add (2.18) and (2.19) to obtain

$$
(1-\tilde{\varepsilon})\left(\rho\left(\tau, u_{0}\right)+\rho\left(u_{0}, \tilde{z}\right)\right)+\rho(t, \tilde{z}) \leq 1 .
$$

But $\rho(t, \tilde{z})<\rho\left(t, u_{0}\right)=\alpha$, since $\tilde{z} \neq u_{0}$ in (2.19); hence $\tilde{z} \notin K_{0}$. Now the previous inequality, (2.21) and $\tilde{\varepsilon} \leq \varepsilon$ show that $\tilde{z}$ satisfies (2.14); therefore it must fail (2.15), i.e.,

$$
\rho(\tau, \tilde{z})>\rho(t, \tilde{z}) .
$$

Also, recall that $u_{0}$ satisfies (2.14) and that we have assumed that (2.14) and (2.16) do not hold simultaneously. This implies that

$$
\rho\left(\tau, u_{0}\right)<1 / 10 \quad \text { or } \quad \rho\left(\tau, u_{0}\right)>9 / 10
$$

and

$$
\rho(\tau, \tilde{z})<1 / 10 \quad \text { or } \quad \rho(\tau, \tilde{z})>9 / 10 .
$$

If $\rho\left(\tau, u_{0}\right)>9 / 10$, then $\rho\left(t, u_{0}\right)>9 / 10$ by (2.15); recall that $u_{0} \in K_{0}$. Then (2.18) furnishes the contradiction

$$
1=(1-\tilde{\varepsilon}) \rho\left(\tau, u_{0}\right)+\rho\left(u_{0}, t\right)>(2-\tilde{\varepsilon}) \frac{9}{10}>1
$$

if, say, $\varepsilon \leq 1 / 4$. The conclusion at this point is

$$
\rho\left(\tau, u_{0}\right)<1 / 10 .
$$

On the other hand, if $\rho(\tau, \tilde{z})<1 / 10$, then $\rho(t, \tilde{z})>9 / 10$ by the triangle inequality, which contradicts (2.22). Consequently

$$
\rho(\tau, \tilde{z})>9 / 10 \text {. }
$$

If we now use that $\tilde{z}$ satisfies (2.19) and (2.20), we derive, for $\varepsilon \leq 1 / 4$, that

$$
\rho\left(u_{0}, \tilde{z}\right) \leq \rho(t, \tilde{z}) \leq 1-(1-\varepsilon) \rho(\tau, \tilde{z}) \leq \frac{13}{40}
$$


and hence the contradiction

$$
\rho(\tau, t) \leq \rho\left(\tau, u_{0}\right)+\rho\left(u_{0}, \tilde{z}\right)+\rho(\tilde{z}, t)<1 .
$$

This completes the proof of the remark.

We do not know any example of a compact space with $(Z)$ that is not metrically convex.

\section{Locality and the Daugavet property}

We can now prove a sufficient criterion for $\operatorname{Lip}(K)$ to have the Daugavet property. In particular it turns out that for closed convex subsets of Banach spaces $\operatorname{Lip}(K)$ has the Daugavet property.

THEOREM 3.1. If $K$ is a spreadingly local metric space (in particular if $K$ is a metrically convex metric space or a compact local metric space), then $\operatorname{Lip}(K)$ has the Daugavet property.

Proof. For short write $X=\operatorname{Lip}(K)$. Due to Lemma 1.1 it is sufficient to prove that for every $\varepsilon \in(0,1 / 4]$, and for every $f, g \in S_{X}$ the closed convex hull of the set $W=\left\{u \in(1+\varepsilon) B_{X}:\|f+u\| \geq 2-\varepsilon\right\}$ contains $g$.

In order to do this fix an $n \in \mathrm{N}$ and select $\varepsilon / 2$-points $s_{1}, \ldots, s_{n}$ of $f$. Let $r \in(0, \varepsilon / 4]$ be a number so small that the balls $U_{i}=B_{K}\left(s_{i}, r\right), i=1, \ldots, n$, are disjoint. Fix a $\delta<r^{2} / 16$, and select $t_{i}, \tau_{i} \in B_{K}\left(s_{i}, \delta\right)$ such that

$$
f\left(\tau_{i}\right)-f\left(t_{i}\right)>(1-\varepsilon / 2) \rho\left(t_{i}, \tau_{i}\right) .
$$

Consider $K_{i}=\left(K \backslash U_{i}\right) \sqcup\left\{t_{i}, \tau_{i}\right\}$ as a subspace of the metric space $K$. Define $u_{i}: K_{i} \rightarrow \mathrm{R}$ as follows: $u_{i}\left(t_{i}\right)=g\left(t_{i}\right), u_{i}\left(\tau_{i}\right)=g\left(t_{i}\right)+f\left(\tau_{i}\right)-f\left(t_{i}\right)$ and $u_{i}(s)=g(s)$ on the rest of $K_{i}$. It follows from Lemma 2.5 that $u_{i}$ satisfies a Lipschitz condition on $K_{i}$ with the constant $1+r / 2<1+\varepsilon / 2$. Extend $u_{i}$ to a function on $K$ preserving the Lipschitz constant, still denoted by $u_{i}$.

Note that each $u_{i}$ belongs to $W$. In fact $\left\|u_{i}\right\| \leq 1+\varepsilon$ by construction and

$$
\left\|f+u_{i}\right\| \geq \frac{\left(f+u_{i}\right)\left(\tau_{i}\right)-\left(f+u_{i}\right)\left(t_{i}\right)}{\rho\left(\tau_{i}, t_{i}\right)}=2 \frac{f\left(\tau_{i}\right)-f\left(t_{i}\right)}{\rho\left(\tau_{i}, t_{i}\right)}>2-\varepsilon .
$$

On the other hand the arithmetic mean of the $u_{i}$ (the simplest convex combination) approximates $\mathrm{g}$, for

$$
\left\|g-\frac{1}{n} \sum_{i=1}^{n} u_{i}\right\|=\frac{1}{n}\left\|\sum_{i=1}^{n}\left(u_{i}-g\right)\right\| \leq \frac{4+2 \varepsilon}{n} .
$$


The last inequality follows from the fact that each $u_{i}-g$ has norm $\leq\left\|u_{i}\right\|+$ $\|g\| \leq 2+\varepsilon$ and their supports $U_{i}$ are disjoint.

Finally we address the question in how far our locality conditions are necessary for the Daugavet property; for compact spaces, this will turn out to be the case (Theorem 3.3 below). The bulk of the technical work will be done in the following lemma.

Lemma 3.2. Suppose $\operatorname{Lip}(K)$ has the Daugavet property. Then for every $t_{1}, t_{2} \in K$ with $\rho\left(t_{1}, t_{2}\right)=a>0$, for every $f \in S_{\operatorname{Lip}(K)}$ with $f\left(t_{2}\right)-f\left(t_{1}\right)=a$ (i.e., $f$ attains its norm at the pair $t_{1}, t_{2}$ ) and for every $\varepsilon>0$ there are $\tau_{1}=\tau_{1}(\varepsilon), \tau_{2}=\tau_{2}(\varepsilon) \in K$ with the following properties:

(1) $f\left(\tau_{2}\right)-f\left(\tau_{1}\right) \geq(1-\varepsilon) \rho\left(\tau_{1}, \tau_{2}\right)$;

(2) $\rho\left(t_{1}, \tau_{2}\right)-\rho\left(t_{1}, \tau_{1}\right) \geq(1-\varepsilon) \rho\left(\tau_{1}, \tau_{2}\right)$, $\rho\left(t_{2}, \tau_{1}\right)-\rho\left(t_{2}, \tau_{2}\right) \geq(1-\varepsilon) \rho\left(\tau_{1}, \tau_{2}\right) ;$

(3) $\rho\left(\tau_{1}, \tau_{2}\right) \rightarrow 0$ as $\varepsilon \rightarrow 0$.

Proof. We shall abbreviate $\operatorname{Lip}(K)$ by $X$. Consider the following functions $y_{i} \in X:$

$$
y_{1}=f, \quad y_{2}(t)=\rho\left(t_{1}, t\right), \quad y_{3}(t)=-\rho\left(t_{2}, t\right) .
$$

For all these functions we have

$$
y_{i}\left(t_{2}\right)-y_{i}\left(t_{1}\right)=a, \quad\left\|y_{i}\right\|=1 .
$$

Then the arithmetic mean $y=\left(y_{1}+y_{2}+y_{3}\right) / 3$ is of norm 1 as well. Consider $x^{*} \in X^{*}$, with the action

$$
x^{*}(x)=\frac{1}{a}\left(x\left(t_{2}\right)-x\left(t_{1}\right)\right) .
$$

Clearly $\left\|x^{*}\right\|=1$. Due to the Daugavet property of $X$ there is, by Lemma 1.1, an $x \in S_{X}$ such that $x^{*}(x)>1-\varepsilon$, i.e.,

$$
x\left(t_{2}\right)-x\left(t_{1}\right)>(1-\varepsilon) a,
$$

and at the same time $\|x-y\|>2-\varepsilon / 3$. The last condition means that there are two distinct points $\tau_{1}, \tau_{2} \in K$ for which

$$
(x-y)\left(\tau_{1}\right)-(x-y)\left(\tau_{2}\right)>(2-\varepsilon / 3) \rho\left(\tau_{1}, \tau_{2}\right),
$$

i.e.,

$$
\frac{1}{3} \sum_{i=1}^{3}\left(\left(x-y_{i}\right)\left(\tau_{1}\right)-\left(x-y_{i}\right)\left(\tau_{2}\right)\right)>(2-\varepsilon / 3) \rho\left(\tau_{1}, \tau_{2}\right) .
$$


Since neither of these three summands exceeds $2 \rho\left(\tau_{1}, \tau_{2}\right)$, we get the following three inequalities:

$$
\left(x-y_{i}\right)\left(\tau_{1}\right)-\left(x-y_{i}\right)\left(\tau_{2}\right)>(2-\varepsilon) \rho\left(\tau_{1}, \tau_{2}\right), \quad i=1,2,3 .
$$

Taking into account $x\left(\tau_{1}\right)-x\left(\tau_{2}\right) \leq \rho\left(\tau_{1}, \tau_{2}\right)$ we deduce that

$$
y_{i}\left(\tau_{2}\right)-y_{i}\left(\tau_{1}\right)>(1-\varepsilon) \rho\left(\tau_{1}, \tau_{2}\right), \quad i=1,2,3 .
$$

The case $i=1$ gives us the requested property (1), and the cases $i=2,3$ of (3.6) immediately provide property (2). Finally, substituting the Lipschitz conditions $x\left(\tau_{1}\right) \leq x\left(t_{1}\right)+\rho\left(t_{1}, \tau_{1}\right)$ and $x\left(\tau_{2}\right) \geq x\left(t_{2}\right)-\rho\left(t_{2}, \tau_{2}\right)$ into (3.5) and applying (3.4) we obtain

$$
\begin{aligned}
(2-\varepsilon) \rho\left(\tau_{1}, \tau_{2}\right) & <x\left(t_{1}\right)-x\left(t_{2}\right)+\rho\left(t_{1}, \tau_{1}\right)+\rho\left(t_{2}, \tau_{2}\right)+y_{i}\left(\tau_{2}\right)-y_{i}\left(\tau_{1}\right) \\
& \leq-(1-\varepsilon) \rho\left(t_{1}, t_{2}\right)+\rho\left(t_{1}, \tau_{1}\right)+\rho\left(t_{2}, \tau_{2}\right)+\rho\left(\tau_{1}, \tau_{2}\right),
\end{aligned}
$$

so

$$
\begin{aligned}
(1-\varepsilon) \rho\left(t_{1}, t_{2}\right) & <\rho\left(t_{1}, \tau_{1}\right)+\rho\left(t_{2}, \tau_{2}\right)-(1-\varepsilon) \rho\left(\tau_{1}, \tau_{2}\right) \\
& \leq(2-\varepsilon)\left(\rho\left(t_{1}, \tau_{1}\right)+\rho\left(t_{2}, \tau_{2}\right)\right)-(1-\varepsilon) \rho\left(t_{1}, t_{2}\right)
\end{aligned}
$$

by the triangle inequality; hence

$$
2 \rho\left(t_{1}, \tau_{1}\right)+2 \rho\left(t_{2}, \tau_{2}\right)>4(1-\varepsilon) /(2-\varepsilon) \rho\left(t_{1}, t_{2}\right) .
$$

Adding to this inequality both inequalities from property (2) we obtain

$$
\begin{aligned}
\rho\left(t_{1}, \tau_{1}\right)+\rho\left(t_{2}, \tau_{2}\right)+ & \rho\left(t_{1}, \tau_{2}\right)+\rho\left(t_{2}, \tau_{1}\right) \\
& \geq 4(1-\varepsilon) /(2-\varepsilon) \rho\left(t_{1}, t_{2}\right)+2(1-\varepsilon) \rho\left(\tau_{1}, \tau_{2}\right) .
\end{aligned}
$$

Since the left hand side is not greater than $2 \rho\left(t_{1}, t_{2}\right)$ we deduce

$$
2(1-\varepsilon) \rho\left(\tau_{1}, \tau_{2}\right) \leq\left(2-4 \frac{1-\varepsilon}{2-\varepsilon}\right) \rho\left(t_{1}, t_{2}\right)
$$

which gives property (3).

We can now deduce the main theorem of this paper.

THEOREM 3.3. If $K$ is a compact metric space, then $\operatorname{Lip}(K)$ has the Daugavet property if and only if $K$ is local.

Proof. The "if" part has already been proved in Theorem 3.1. Let us prove the "only if" part. Assume $K$ is not local. Then there is a function $f \in \operatorname{Lip}(K)$, $\|f\|=1$, and there is an $r>0$ such that

$$
f\left(\tau_{2}\right)-f\left(\tau_{1}\right)<(1-r) \rho\left(\tau_{1}, \tau_{2}\right)
$$


for every $\tau_{1}, \tau_{2} \in K$ with $\rho\left(\tau_{1}, \tau_{2}\right)<r$. Hence by a compactness argument there is a pair of points $t_{1}, t_{2} \in K$ with $\rho\left(t_{1}, t_{2}\right)>0$ on which $f$ attains its norm, i.e., with $f\left(t_{2}\right)-f\left(t_{1}\right)=\rho\left(t_{1}, t_{2}\right)$. If nevertheless $\operatorname{Lip}(K)$ has the Daugavet property, then applying Lemma 3.2 to $f$ and these $t_{1}, t_{2}$ with $\varepsilon \rightarrow 0$ entails a contradiction between (3.7) and properties (1) and (3) from the lemma.

The space $\operatorname{Lip}(K)$ has a canonical predual, called the Arens-Eells space in [13] and the Lipschitz free space in [4] and [7]. Since we have used in (3.3), in the proof of Lemma 3.2, a functional from that predual, i.e., a weak* open slice, the lemma works under the assumption that the Lipschitz free space on $K$ has the Daugavet property. Consequently, for a compact metric space $\operatorname{Lip}(K)$ has the Daugavet property if and only if its Lipschitz free space has.

In the setting of subsets of certain Banach spaces like $L_{p}, 1<p<\infty$, we can rephrase Theorem 3.3 as follows, using Corollary 2.10.

COROLlaRY 3.4. If $K$ is a compact subset of a smooth locally uniformly rotund Banach space, then $\operatorname{Lip}(K)$ has the Daugavet property if and only if $K$ is convex.

\section{REFERENCES}

1. Benyamini, Y., and Lindenstrauss, J., Geometric Nonlinear Functional Analysis, Vol. 1, Amer. Math. Soc. Colloq. Publ. 48, 2000.

2. Bilik, D., Kadets, V., Shvidkoy, R., and Werner, D., Narrow operators and the Daugavet property for ultraproducts, Positivity 9 (2005), 46-62.

3. Foiaş, C., and Singer, I., Points of diffusion of linear operators and almost diffuse operators in spaces of continuous functions, Math. Z. 87 (1965), 434-450.

4. Godefroy, G., and Kalton, N., Lipschitz-free Banach spaces, Studia Math. 159 (2003), 121141.

5. Kadets,V. M., Shvidkoy, R. V., Sirotkin, G. G., and Werner, D., Banach spaces with the Daugavet property, Trans. Amer. Math. Soc. 352 (2000), 855-873.

6. Kadets, V. M., Shvidkoy, R. V., and Werner, D., Narrow operators and rich subspaces of Banach spaces with the Daugavet property, Studia Math. 147 (2001), 269-298.

7. Kalton, N., Spaces of Lipschitz and Hölder functions and their applications, Collect. Math. 55 (2004), 171-217.

8. Oikhberg, T., The Daugavet property of $C^{*}$-algebras and non-commutative $L_{p}$-spaces, Positivity 6 (2002), 59-73.

9. Rudin, W., Functional Analysis, McGraw-Hill, 1973.

10. Shvidkoy, R. V., Geometric aspects of the Daugavet property, J. Funct. Anal. 176 (2000), 198-212.

11. Singer, I., Best Approximation in Normed Linear Spaces by Elements of Linear Subspaces, Springer, 1970.

12. Vlasov, L. P., Chebyshev sets in Banach spaces, Soviet Math. Dokl. 2 (1962), 1373-1374.

13. Weaver, N., Lipschitz Algebras, World Scientific, 1999.

14. Werner, D., The Daugavet equation for operators on function spaces, J. Funct. Anal. 143 (1997), 117-128. 
15. Werner, D., Recent progress on the Daugavet property, Irish Math. Soc. Bull. 46 (2001), 77-97.

16. Wojtaszczyk, P., Some remarks on the Daugavet equation, Proc. Amer. Math. Soc. 115 (1992), 1047-1052.

FACULTY OF MECHANICS AND MATHEMATICS

KHARKOV NATIONAL UNIVERSITY,

PL. SVOBODY 4

61077 KHARKOV

UKRAINE

E-mail: ivakhnoj@yandex.ru,vova1kadets@yahoo.com
DEPARTMENT OF MATHEMATICS

FREIE UNIVERSITÄT BERLIN

ARNIMALLEE 2-6

D-14 195 BERLIN

GERMANY

E-mail: werner@math.fu-berlin.de 